\title{
QUASICONVEX FUNCTIONS AND HESSIAN EQUATIONS
}

\author{
DANIEL FARACO AND XIAO ZHONG
}

\begin{abstract}
In this note we construct new examples of quasiconvex functions defined on the set $\mathbb{S}^{n \times n}$ of symmetric matrices. They are built on the $k$-th elementary symmetric function of the eigenvalues, $k=1,2, \ldots, n$. The idea is motivated by Šverák's paper [S]. The proof of our result relies on the theory of the so-called k-Hessian equations, which have been intensively studied recently, see [CNS], [T], [TW1], [TW2].
\end{abstract}

\section{INTRODUCTION}

The purpose of this note is to bring together the theory of k-Hessian equations with that of quasiconvex functions and Gradient Young measures. Quasiconvexity has turned out to be the right notion to characterize the lower semicontinuity of functionals in the vectorial calculus of variations $[\mathrm{M}]$. Unfortunately, it is very hard to check if a function is quasiconvex or not from its definition, cf (2.1). In [B], Ball introduced the notion of polyconvexity which in particular implies quasiconvexity. However the reverse implication is not true and often polyconvexity is too general. Therefore there is a pressing need for quasiconvex functions which are not polyconvex.

In [S], Šverák constructed examples of quasiconvex functions restricted to symmetric matrices by truncating the determinant. These examples have turned out to be very useful in a number of different problems where polyconvexity did not suffice, e.g [D], [DKMS], [K], [MüS],[S].

Motivated by [S], we provide more quasiconvex functions by truncating the sum of the $k \times k$ principal minors. To state the definition, we briefly introduce the Hessian equations. Caffareli, Nirenberg and Spruck considered in [CNS] a class of nonlinear second order elliptic equations, and obtained the classical existence result. The operators they considered are defined by certain smooth symmetric functions of the eigenvalues of the Hessian matrices. A prime example of these

\footnotetext{
Key words and phrases. quasiconvex funcions, Young measures, $k$-Hessian equations.

2000 Mathematics Subject Classification 35J60 ,49J40, 49J45, 52A30.

X.Z. is supported by the Academy of Finland (Projects \#51947 and \#41933).

D.F. is supported by the Academy of Finland(Project \# 53292).
} 
operators is the so called $k$-Hessian operator, which has been extensively studied recently, see [T], [TW1], [TW2], [TW3] and the references therein. Let $\Omega \subset \mathbf{R}^{n}$ be an open set. For $k=1,2, \ldots, n$ and a function $u \in C^{2}(\Omega)$, the k-Hessian operator is defined by

$$
F_{k}(u)=S_{k}\left(\lambda\left(D^{2} u\right)\right),
$$

where $D^{2} u$ denotes the Hessian matrix of the second derivatives of $u$, $\lambda(A)=\left(\lambda_{1}, \lambda_{2}, \ldots, \lambda_{n}\right)$ the vector of eigenvalues of an $n \times n$ matrix $A$ and $S_{k}(\lambda)$ the $k$-th elementary symmetric function on $\mathbf{R}^{n}$, given by

$$
S_{k}(\lambda)=\sum_{i_{1}<\ldots<i_{k}} \lambda_{i_{1}} \ldots \lambda_{i_{k}}
$$

Alternatively, we may write

$$
F_{k}(u)=\left[D^{2} u\right]_{k},
$$

where $[A]_{k}$ denotes the sum of the $k \times k$ principal minors of an $n \times$ $n$ matrix $A \in \mathbb{M}^{n \times n}$. We notice that $F_{1}(u)=\Delta u$ is the Laplacian operator and $F_{n}(u)=\operatorname{det}\left(D^{2} u\right)$ the Monge-Ampére operator.

A function $u \in C^{2}(\Omega)$ is $k$-convex in $\Omega$ if $F_{j}(u) \geq 0$ in $\Omega$ for $j=$ $1,2, \ldots, k$; that is, the eigenvalues $\lambda\left(D^{2} u\right)$ of the Hessian $D^{2} u$ of $u$ lie in the closed convex cone given by

$$
\Gamma_{k}=\left\{\lambda \in \mathbf{R}^{n}: S_{j}(\lambda) \geq 0, j=1,2, \ldots, k\right\},
$$

see section 2 for the basic properties of $\Gamma_{k}$. Now we are ready to define our functions. Let $A \in \mathbb{S}^{n \times n}$, the set of $n \times n$ symmetric matrices. We define for $k=1,2, \ldots, n$

$$
G_{k}(A)= \begin{cases}{[A]_{k},} & \text { if } \lambda(A) \in \Gamma_{k} \\ 0, & \text { otherwise }\end{cases}
$$

Theorem 1.1. Let $k=1,2, \ldots, n$. The function $G_{k}$ defined above is quasiconvex on $\mathbb{S}^{n \times n}$.

We note that

$$
G_{1}(A)=\max \left(F_{1}(A), 0\right)=\max (\operatorname{trace}(A), 0) .
$$

Thus Theorem 1.1 is trivial when $k=1$. The case $k=n$ was treated in $[\mathrm{S}]$.

Šverák used the Hodge decomposition to prove that Gradient Young measures supported in the set of symmetric matrices satisfy Jensen's inequality for quasiconvex functions on the symmetric matrices [S, Lemma 1]. This yields the following corollary of Theorem 1.1. For the definition and usefulness of Gradient Young measures see for example [Mü], $[\mathrm{P}]$ and the references therein. 
Corollary 1.2. Let $\{\nu\}_{x \in \Omega}$ be $W^{1, p}$-Gradient Young measure for $p \geq k$. Then for a.e. $x \in \Omega$

$$
G_{k}\left(\int_{\mathbb{S}^{n \times n}} A d \nu_{x}(A)\right) \leq \int_{\mathbb{S}^{n \times n}} G_{k}(A) d \nu_{x}(A) .
$$

The corollary yields immediately the following proposition.

Proposition 1.3. Let $\nu$ be an homogeneous $W^{1, p}$-Gradient Young measure for $p \geq k$ supported on the set $\left\{A \in \mathbb{S}^{n \times n}:[A]_{k} \geq \epsilon\right\}$ for some $\epsilon>0$. Suppose further that the center of mass of $\nu$ belongs to $\Gamma_{k}$. Then $\nu$ is supported in $\Gamma_{k}$.

Šverák's proof of Theorem 1.1 in the case $k=n$ is based on the fact that we understand the meaning of the measure induced by $G_{n}\left(D^{2} u\right)$. Briefly, for a Borel set $E, G_{n}\left(D^{2} u\right)(E)=\left|\nabla u\left(E \cap \Gamma_{u}\right)\right|$, where $\Gamma_{u}$ is the subset of $\Omega$ where $u$ is convex. However the $k$-Hessian measures do not have such a clear interpretation and thus it is not clear for us how to extend Šverák proof to our case. Therefore, our approach is essentially different and naturally it yields a new proof of Šverák's result. Our argument to deal with Theorem 1.1 relies on the theory of $k$-Hessian equations. The outline of the proof is as follows. To show that $G_{k}$ is quasiconvex, we need to show that

$$
\int_{\Omega} G_{k}(A) d x \leq \int_{\Omega} G_{k}\left(A+D^{2} \varphi\right) d x
$$

for each $A \in \mathbb{S}^{n \times n}$ and each $\varphi \in C_{0}^{\infty}(\Omega)$. The critical point is to solve the following $k$-Hessian equation

$$
\begin{cases}F_{k}(u)=G_{k}\left(A+D^{2} \varphi\right) & \text { in } \Omega \\ u=\frac{1}{2}\langle A x, x\rangle+\varphi & \text { on } \partial \Omega .\end{cases}
$$

Actually, a modification of $G_{k}\left(A+D^{2} \varphi\right)$ on the right hand side is needed so that the solution $u$ is smooth. The existence of solution is guaranteed by [CNS], see also [T]. Then the comparison principle Lemma 2.3 shows that

$$
u \leq \frac{1}{2}\langle A x, x\rangle+\varphi \quad \text { in } \Omega
$$

Thus outside of the support of $\varphi$, the k-convex function $u$ is below the function $v=\frac{1}{2}\langle A x, x\rangle$, which can be assumed to be $k$-convex, since otherwise (1.4) is trivial. Now Lemma 2.4 implies that

$$
\int_{\Omega} F_{k}(v) d x \leq \int_{\Omega} F_{k}(u) d x
$$

which is exactly (1.4) because of (1.5) and hence the proof is concluded.

The details of the proof of Theorem 1.1 are given in section 3 after some notation and preliminary results in section 2 . 
Acknowledgments. This work was inspired by a seminar on Hessians Equations at the University of Jyväskylä. We would like to thank Petri Juutinen, Tero Kilpeläinen and all the other participants for making the seminar possible and for stimulating and helpful discussions.

\section{Notations AND PRELIMINARY RESUlts}

Through this paper, $\Omega$ denotes a bounded open subset of $\mathbf{R}^{n}, \mathbb{M}^{n \times n}$ the set of all $n \times n$ matrices and $\mathbb{S}^{n \times n} \subset \mathbb{M}^{n \times n}$ the subspace of all symmetric matrices. We recall that a continuous function $f: \mathbb{M}^{n \times n} \rightarrow$ $\mathbf{R}$ is said to be quasiconvex if

$$
\int_{\Omega} f(A) d x \leq \int_{\Omega} f(A+D \varphi) d x .
$$

for any matrix $A \in \mathbb{M}^{n \times n}$ and any smooth $\varphi: \Omega \rightarrow \mathbf{R}^{n}$ compactly supported in $\Omega$.

A continuous function $f: \mathbb{S}^{n \times n} \rightarrow \mathbf{R}$ is said to be quasiconvex if

$$
\int_{\Omega} f(A) \leq \int_{\Omega} f\left(A+D^{2} \varphi\right) d x
$$

for any $A \in \mathbb{S}^{n \times n}$ and any smooth function $\varphi: \Omega \rightarrow \mathbf{R}$ compactly supported in $\Omega$. It turns out that the domain $\Omega$ plays no role in the definition of quasiconvexity; if $f$ satisfies condition (2.1) or (2.2) respect to one smooth domain, it satisfies them for every smooth domain, see $[\mathrm{M}]$.

The remaining of the section is devoted to present some basic properties of the $k$-th elementary symmetric function $S_{k}$ and the cone $\Gamma_{k}$ and the results about k-Hessian equations, which are needed in the next section.

Recall that the definition of $S_{k}$ is

$$
S_{k}(\lambda)=\sum_{i_{1}<\ldots<i_{k}} \lambda_{i_{1}} \ldots \lambda_{i_{k}}
$$

Let $\Gamma_{+}=\left\{\eta \in \mathbf{R}^{n}: \eta_{j} \geq 0, j=1, \ldots, n\right\}$. Then, we have the following alternative characterizations for the cone $\Gamma_{k}$.

\section{Proposition 2.1.}

$$
\begin{aligned}
\Gamma_{k} & =\left\{\lambda \in \mathbf{R}^{n}: S_{j}(\lambda) \geq 0 \quad \text { for } j=1,2, \ldots, k\right\} \\
& =\left\{\lambda \in \mathbf{R}^{n}: 0 \leq S_{k}(\lambda) \leq S_{k}(\lambda+\eta) \quad \text { for } \eta \in \Gamma_{+}\right\} \\
& =\left\{\lambda \in \mathbf{R}^{n}: S_{k}(\lambda+\eta) \geq 0 \quad \text { for } \eta \in \Gamma_{+}\right\} \\
& =\text {The closure of the component of } S_{k}^{-1}(0, \infty) \text { containing } \Gamma_{+} .
\end{aligned}
$$

We note here that $\Gamma_{n}=\Gamma_{+}$. A fundamental property of $\Gamma_{k}$ is that it is convex. The function $S_{k}^{\frac{1}{k}}$ is concave in $\Gamma_{k}$. See [CNS] or [TW2] for the proof of these facts. 
Then if we consider the function $[A]_{k}=S_{k}(\lambda(A)),[A]_{k}^{\frac{1}{k}}$ is a concave function on the convex set

$$
M_{k}=\left\{A \in \mathbb{S}^{n \times n}: \lambda(A) \in \Gamma_{k}\right\}
$$

This is not trivial. We refer to $[\mathrm{B}]$ or $[\mathrm{CNS}]$ for the proof.

Recall that a function $u \in C^{2}(\Omega)$ is k-convex if $\lambda\left(D^{2} u\right)$ lies in $\Gamma_{k}$. Denote the set of $k$-convex functions in $\Omega$ by $\Psi^{k}(\Omega)$. We refer to the series of papers [TW1], [TW2], [TW3] for an extensive study of the k-convex functions. The following fundamental theorems are needed for our approach to work.

Theorem 2.2. [CNS, Theorem 2] Let $\Omega$ be a bounded, uniformly $(k-$ $1)$-convex domain in $\mathbf{R}^{n}$ with the boundary $\partial \Omega \in C^{\infty}$ and $\varphi$, $\psi$ be functions in $C^{\infty}(\bar{\Omega})$ with $\inf _{\Omega} \psi>0$. Then there exists a unique $k$ convex function $u \in C^{\infty}(\bar{\Omega})$ solving the Dirichlet problem

$$
\begin{cases}F_{k}(u)=\psi & \text { in } \Omega \\ u=\varphi & \text { on } \partial \Omega\end{cases}
$$

In addition we need the following comparison principle. The result is a special case of Lemma 2.1 in [T]. Since the proof is simple in our situation, we include it for the reader's convenience.

Lemma 2.3. Let $u, v \in C^{0}(\bar{\Omega}) \cap C^{2}(\Omega)$ satisfy that $u \leq v$ on $\partial \Omega$ and that $u$ is $k$-convex in $\Omega$. Suppose moreover that whenever $v-u$ is $k$-convex,

$$
\left[D^{2} u\right]_{k}>\left[D^{2} v\right]_{k}
$$

Then $u \leq v$ in $\Omega$.

Proof. Consider the function $w=v-u$. Since $w \geq 0$ on $\partial \Omega$, if there exists a point inside $\Omega$ such that $w<0$, the function has a local minimum at a point $x_{0} \in \Omega$. Therefore $D^{2}(w)\left(x_{0}\right)$ is positive semidefinite. In particular, the function $w$ is $k$-convex at $x_{0}$.

Consider the function $f(A)=[A]_{k}^{\frac{1}{k}}$. Then $f(A)$ is homogeneous of degree 1 and concave on the convex cone $M_{k}$, the set of matrices having eigenvalues in $\Gamma_{k}$. Moreover, by the convexity of $M_{k}$, the sum of two $k$-convex functions is always $k$-convex. Thus $v=w+u$ is $k$-convex at $x_{0}$, since $u$ is k-convex in $\Omega$ and $w$ is also $k$-convex at $x_{0}$. Now $\frac{1}{2} v=\frac{1}{2} w+\frac{1}{2} u$. Since $f$ is concave,

$$
f\left(D^{2} \frac{1}{2} v\left(x_{0}\right)\right) \geq \frac{1}{2} f\left(D^{2} w\left(x_{0}\right)\right)+\frac{1}{2} f\left(D^{2} u\left(x_{0}\right)\right) .
$$

Using the homogeneity of $f$ and rearranging, we arrive at

$$
f\left(D^{2} w\left(x_{0}\right)\right) \leq f\left(D^{2} v\left(x_{0}\right)\right)-f\left(D^{2} u\left(x_{0}\right)\right)<0,
$$

by the assumption of the lemma. This yields a contradiction. 
Finally, we need the following monotonicity lemma, which is stated in a weaker form in Lemma 2.1 of [TW1]. Our proof is based on theirs but it seems we get rid of some of the technical parts.

Lemma 2.4. [TW1, Lemma 2.1] Let $\Omega \in \mathbf{R}^{n}$ be a bounded smooth domain. Suppose that $u, v \in \Psi(\Omega) \cap C^{2}(\bar{\Omega})$ satisfy that $u=v$ on $\partial \Omega$ and that $u \geq v$ in $\Omega_{\delta}=\{x \in \Omega: \operatorname{dist}(x, \partial \Omega) \leq \delta\}$ for some $\delta>0$. Then

$$
\int_{\Omega} F_{k}(u) \leq \int_{\Omega} F_{k}(v)
$$

For the proof, we need some notations and results. For a matrix $A \in \mathbb{S}^{n \times n},(A)_{i j}=a_{i j}$, we use the notation

$$
D\left([A]_{k}\right)=A_{k}^{\#},
$$

where $\left(A_{k}^{\#}\right)_{i j}=\partial a_{i j}[A]_{k}$. Let $f \in C^{2}\left(\Omega, \mathbf{R}^{n}\right)$. Then since $[A]_{k}$ is a null-Lagrangian, we have that

$$
\operatorname{Div}\left((D f(x))_{k}^{\#}\right)=0,
$$

i.e $\partial_{j}\left((D f(x))_{k}^{\#}\right)_{i j}=0 \forall i=1, \ldots, n$, see $[\mathrm{R}]$. On the other hand, the $k$-Hessian operator is degenerate elliptic when acting on $k$-convex functions. In turn this implies that $A_{k}^{\#} \geq 0$ when $A$ is a symmetric matrix with $\lambda(A) \in \Gamma_{k}$, see [TW2].

Proof. In the proof we use $\langle\cdot, \cdot\rangle$ to denote the inner product of both $\mathbf{R}^{\mathbf{n}}$ and $\mathbb{M}^{n \times n}$. Let $\gamma$ denote the outer unit normal to $\partial \Omega$ and $\partial$ the tangential gradient in $\partial \Omega$. That is,

$$
\partial=(I-\gamma \otimes \gamma) \nabla
$$

From the hypotheses in the lemma, we have that on $\partial \Omega$

$$
\partial(u-v)=0, \quad\langle\gamma, \nabla(u-v)\rangle \leq 0 .
$$

we write as in [TW1]

$$
\left.\int_{\Omega} F_{k}(v)-F_{k}(u)\right)=\int_{0}^{1} \int_{\Omega}\left\langle\left(s D^{2} u+(1-s) D^{2} v\right)_{k}^{\#}, D^{2}(v-u)\right\rangle .
$$

By (2.5) and the divergence theorem, the right side of the above equality equals to

$$
\int_{0}^{1} \int_{\partial \Omega}\left\langle\left(s D^{2} u+(1-s) D^{2} v\right)_{k}^{\#} \gamma, \nabla(v-u)\right\rangle .
$$

Now we use our assumptions. Let $A$ be any nonnegative symmetric matrix. Then $\partial(v-u)=0$ implies that

$$
A(I-\gamma \otimes \gamma) \nabla(v-u)=0 \text {, }
$$

that is,

$$
A \nabla(v-u)=A \gamma\langle\gamma, \nabla(v-u)\rangle .
$$


Hence

$$
\langle A \gamma, \nabla(v-u)\rangle=\langle A \gamma, \gamma\rangle\langle\gamma, \nabla(v-u)\rangle \geq 0
$$

where we have used (2.6) and that $A$ is nonnegative. Taking $A$ to be $\left(s D^{2} u+(1-s) D^{2} v\right)_{k}^{\#}$ in $(2.7)$ and taking account of the $k$-convexity of $s u+(1-s) v$, we conclude the proof.

\section{Proof of Theorem 1.1}

We need to prove that

$$
\int_{\Omega} G_{k}(A) d x \leq \int_{\Omega} G_{k}\left(A+D^{2} \varphi\right) d x
$$

for any $A \in \mathbb{S}^{n \times n}$ and $\varphi \in C_{0}^{\infty}(\Omega)$ where $G_{k}$ is defined as in (1.2). Since the definition of quasiconvexity is independent of the domain we can assume that $\Omega$ is the unit Ball $B_{1}$. We can also assume that $\lambda(A) \in \Gamma_{k}$, since otherwise the inequality is trivial.

Let $v=\frac{1}{2}\langle A x, x\rangle+\varphi$, where $\varphi \in C_{0}^{\infty}\left(B_{1}\right)$. Let $r<1$ be such that $\operatorname{supp}(\varphi) \subset B_{r}$. We note that the regularization of the function $G_{k}\left(D^{2} v\right)$ with a mollifier $\rho,\left(G_{k}\left(D^{2} v\right)_{h}=\rho_{h} * G_{k}\left(D^{2} v\right)\right.$ converges uniformly to $G_{k}\left(D^{2} v\right)$ in $B_{\frac{1+r}{2}}$ as $h \rightarrow 0$, since $G_{k}\left(D^{2} v\right)$ is a continuous function in $B_{1}$. Then for any $\epsilon$, there is $0<h_{0}<\frac{R-r}{4}$ such that for any $h<h_{0}$, we have

$$
\left\|\left(G_{k}\left(D^{2} v\right)\right)_{h}-G_{k}\left(D^{2} v\right)\right\|_{C\left(\overline{B_{\frac{1+r}{2}}}\right)} \leq \frac{\epsilon}{2} .
$$

By Theorem 2.2, the following Dirichlet problem

$$
\begin{cases}F_{k}(u)=\left(G_{k}\left(D^{2} v\right)\right)_{h}+\epsilon & \text { in } B_{\frac{1+r}{2}} \\ u=v & \text { on } \partial B_{\frac{1+r}{2}}\end{cases}
$$

has a unique solution $u \in C^{\infty}\left(\overline{B_{\frac{1+r}{2}}}\right) \cap \Psi^{k}\left(B_{\frac{1+r}{2}}\right)$. Note that by (3.2),

$$
\left(G_{k}\left(D^{2} v\right)\right)_{h}+\epsilon \geq G_{k}\left(D^{2} v\right)+\frac{\epsilon}{2} \geq \frac{\epsilon}{2} .
$$

Now we want to apply Lemma 2.3. Suppose that $v-u$ is $k$-convex at a point $x_{0}$. Then $v=v-u+u$ is also $k$-convex at $x_{0}$. Thus at $x_{0}$, $G_{k}\left(D^{2} v\right)=F_{k}(v)$ by the definition of the function $G_{k}$. Therefore it follows from (3.4) that

$$
F_{k}(u)>F_{k}(v)
$$

on the set where $v-u$ is $k$-convex. Applying Lemma 2.3 to $u$ and $v$, we obtain that $u \leq v$ in $B_{\frac{1+r}{2}}$. We explicitly remark here that $v$ coincides with the function $\frac{1}{2}\langle A x, x\rangle$ outside the ball $B_{r}$. Now we are in the position to use lemma 2.4 for the two smooth $k$-convex functions $u$ and $\frac{1}{2}\langle A x, x\rangle$ with $\Omega=B_{\frac{1+r}{2}}$. Then we have that

$$
\int_{B_{\frac{1+r}{2}}} G_{k}(A) d x=\int_{B_{\frac{1+r}{2}}} F_{k}\left(\frac{1}{2}\langle A x, x\rangle\right) d x \leq \int_{B_{\frac{1+r}{2}}} F_{k}(u) d x=
$$




$$
=\int_{B_{\frac{1+r}{2}}}\left(G_{k}\left(D^{2} v\right)\right)_{h}+\epsilon d x
$$

The proof is concluded by letting first $h$ go to 0 and then $\epsilon$ to 0 and finally $r$ to 1.

Proof of Proposition 1.3. Following Šverák, we base the proof on the fact that the function $R_{k}(A)=\max \left\{\epsilon+G_{k}(A)-[A]_{k}, 0\right\}$ is quasiconvex on the space of symmetric matrices. Notice that $R_{k}(A)$ is equal to $\epsilon$ if $\lambda(A) \in \Gamma_{k}$ and $R_{k}(A) \leq \epsilon$ everywhere. Using Corollary 1.2 for the measure $\nu$ we have that $\nu\left(\left\{A: \lambda(A) \in \Gamma_{k}\right\}\right)=1$ and the proposition is proved.

Proposition 1.3 and the related work of Šverák [S2] for convex functions and the Monge-Ampére equation suggest the following conjecture for $k$-convexity.

Conjecture. Let $u$ be a $W^{2, \infty}(\Omega)$ be such that $\left[D^{2} u\right]_{k} \geq \epsilon$ and suppose that the set $\left\{x \in \Omega: \lambda\left(D^{2} u\right) \in \Gamma_{k}\right\}$ has positive measure. Then $u$ is $k$-convex.

\section{REFERENCES}

[B] J. M. Ball, Convexity conditions and existence theorems in nonlinear elasticity, Arch. Rational Mech. Anal. 63 (1978), 337-403.

[CNS] L. Caffarelli, L. Nirenberg and J. Spruck, The Dirichlet problem for nonlinear second order elliptic equations III, Functions of the eigenvalues of the Hessian, Acta Math. 155 (1985), 261-301.

[D] G. Dolzmann, Quasiconvex hulls in symmetric matrices. Preprint(2001)

[DKMS] G.Dolzmann, B. Kirchheim, S. Müller, and V. Šverák, The two-well problem in three dimensions. Calc. Var. Partial Differential Equations 10 (2000), no. 1, 21-40.

[K] B, Kirchheim, Geometry and rigidity of microstructures. Habilitation Thesis, Leipzig 2001.

[M] C. B. Morrey, Quasi-convexity and the lower semicontinuity of multiple integrals, Pacific J. Math. 2 (1952), 25-53.

[Mü] S. Müller, Variational models for microstructure and phase transitions. Calculus of variations and geometric evolution problems (Cetraro, 1996), 85-210, Lecture Notes in Math., 1713, Springer, Berlin, 1999.

[MüS] S. Müller and V. Šverák, Convex integration for Lipschitz mappings and counterexamples to regularity. Preprint (1999)

[P] P. Pedregal, Parametrized Measures and Variational principles. Birkhauser, 1997.

[R] R. C. Reilly, On the Hessian of a function and the curvatures of its graph, Michigan Math. J. 20 (1973-74), 373-383.

[S] V. Šverák, New examples of quasiconvex functions, Arch. Rational Mech. Anal. 119 (1992), 293-300.

[S2] V, Šverák. On regularity for the Monge-Ampere Equation without convexity assumptions. Preprint (1991)

[T] N. S. Trudinger, Weak solutions of Hessian equations, Comm. Partial Diff. Eq. 22 (1997), 1251-1261.

[TW1] N. S. Trudinger and X. J. Wang, Hessians measures I, Topol. Math. in Nonlinear Anal. 10 (1997), 225-239. 
[TW2] N. S. Trudinger and X. J. Wang, Hessians measures II, Ann. of Math. 150 (1999), 579-604.

[TW3] N. S. Trudinger and X. J. Wang, Hessians measures III, preprint.

University of Jyväskylä, Department of Mathematics and Statistics P.O. Box 35, FIN-40014 Jyväskylä, Finland

E-mail: danfara@math.jyu.fi_zhong@math.jyu.fi 